\title{
Numerical Capacity Analysis of Time Varying Fading Channels Using Finite State Markov Models
}

\author{
Parastoo Sadeghi ${ }^{1}$ \\ School of Elec. Eng. and Telecomm. \\ The University of New South Wales \\ Sydney NSW 2052 Australia \\ e-mail: parastoo@student.unsw.edu.au
}

\author{
Predrag B. Rapajic ${ }^{1}$ \\ School of Elec. Eng. and Telecomm. \\ The University of New South Wales \\ Sydney NSW 2052 Australia \\ e-mail: p.rapajic@unsw.edu.au
}

Sarah J. Johnson
Wireless Signal Processing Program
National ICT Australia Ltd.
Sydney NSW 2052 Australia
e-mail: sarah.johnson@nicta.com.au

\begin{abstract}
We investigate the effect of channel gain quantization on the information capacity of unknown time varying flat fading channels. The phase and/or amplitude of the flat fading channel gain is modelled as a finite state Markov (FSM) process and the information capacity of the FSM channel is calculated numerically as a measure for choosing the number of channel quantization levels, as well as quantization thresholds. The results indicate that for binary signalling, the capacity is saturated beyond 8 to 16 levels of phase and 8 to 16 levels of amplitude quantization.
\end{abstract}

\section{Extended Summary}

According to the classical communication theory of time invariant additive white Gaussian noise (AWGN) channels, the quantization of the received signal into 4 and 8 levels (softdecision decoding) results in approximately $1.4 \mathrm{~dB}$ and 1.8 $\mathrm{dB}$ performance gain compared to 2-level binary quantization (hard-decision decoding), respectively. Whereas, there is only a $0.2 \mathrm{~dB}$ margin between the receiver performance with 8-level signal quantization and the continuous-level soft-decision decoding limit. A similar question arises in the estimation of the complex channel gain for coherent detection in unknown time varying flat fading channels. Namely, "What is the minimum quantization resolution for estimating the complex channel gain without losing much in the channel capacity?".

Following Clarke's model for flat fading channels, the sampled output of the matched filter at the $n^{t h}$ signalling interval is

$$
Y_{n}=C_{n} X_{n}+Z_{n}=R_{n} e^{j \Theta_{n}} X_{n}+Z_{n} .
$$

where $X_{n}, Y_{n}, Z_{n}$ are the channel input, output, and AWGN, respectively. The signal to noise ratio is denoted by $\gamma_{b}=$ $E_{b} / N_{0}$. The channel fading gain, $C_{n}$, is a zero-mean stationary complex Gaussian process, with auto-correlation function given by $R_{A C F}(m)=\sigma^{2} J_{0}\left(2 \pi m f_{D} T\right)$, where $f_{D}$ is the maximum Doppler frequency and $T$ is the symbol period.

An infinite channel memory order is assumed in the Clarke's model, which is prohibitive in terms of receiver design. Research results indicate that assuming a finite memory order, between 0 to 5 , is justified for accurately tracking the channel dynamics at the receiver side [1]. It is also shown that the first-order memory Markov model is applicable for flat fading channels with $f_{d} T \lesssim 0.01$ [2].

In the literature, the emphasis is often on the quantization and finite state Markov (FSM) mapping [3] of the fading amplitude $R_{n}$. However, since phase estimation is essential for coherent detection, we study joint phase and amplitude quantization and FSM mapping of the complex fading process.

\footnotetext{
${ }^{1}$ This work was supported by ARC Discovery Grant.
}

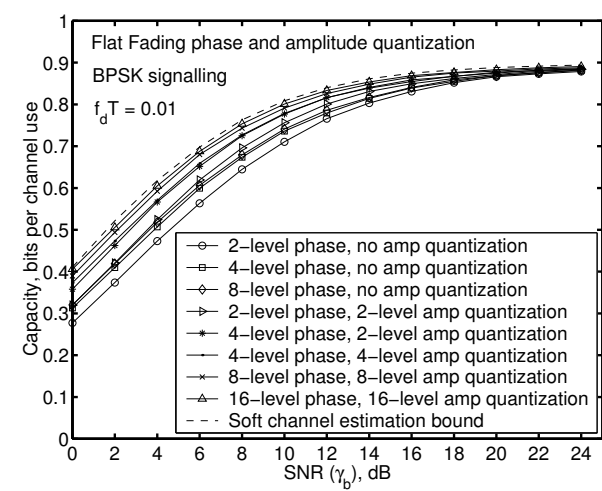

Fig. 1: Capacity as a function of SNR. There is only $0.3 \mathrm{~dB}$ gain in soft channel estimation, compared to 16 -level phase and 16-level amplitude quantization.

We study the information capacity of the quantized flat fading channels and propose the quantization gain as a measure to choose the optimum quantization resolution and threshold for the channel estimation. Therefore, the best tradeoff between computational complexity of handling more channel states and channel quantization gain is obtained.

In Fig. 1, fading amplitude and phase are mapped into a first-order memory Markov model [4] and the capacity is calculated using the numerical method [5], which makes capacity analysis of FSM channels with large number of states practically feasible. The general trend carries over to slower and faster fading conditions, as well. For very slow fading, where channel state information (CSI) assumption is accurate, capacity with 8-level quantized phase and 8-level quantized amplitude CSI is within $0.2 \mathrm{~dB}$ of the continuous-level CSI limit.

\section{ACKNOWLEDGMENTS}

The authors would like to thank Dr. A. Kavcic for bringing the numerical method to their attention and helpful discussions.

\section{REFERENCES}

[1] M. Riediger and E. Shwedyk, "Communication receivers based on Markov models of the fading channel," IEE Proc. Commun. vol. 150 , no. 4, pp. 275-279, Aug. 2003.

[2] F. Babich, O. E. Kelly, and G. Lombardi, "A context-tree based model for quantized fading," IEEE Commun. Lett., vol. 3, no. 2, pp. 46-48, Feb. 1999.

[3] A. J. Goldsmith and P. Varaiya, "Capacity, mutual information, and coding for finite state Markov channels," IEEE Trans. Inform. Theory, vol. 42, no. 3, pp. 868-886, May 1996.

[4] P. Sadeghi and P. Rapajic, "Capacity analysis of time varying fading channels using finite state Markov models," submitted to IEEE Trans. Wireless Commun.

[5] A. Kavcic, "On the capacity of Markov sources over noisy channels", IEEE Global commun. Conf. (GLOBECOM), pp. 29973001, 2001. 